\title{
Comunicação
}

[Communication]

\section{Avaliação por eletrocardiografia contínua (holter) em cães da raça Pastor Alemão praticantes de atividade física regular}

\author{
[Ambulatory electrocardiography (Holter) evaluation in physically active German shepherd dogs] \\ G.A.O. Cavalcanti ${ }^{1}$, R.B. Nogueira ${ }^{2}$, G.R. Sampaio $^{2}$, R.B. Araújo ${ }^{1}$, R.S. Gonçalves ${ }^{3}$ \\ ${ }^{1}$ Escola de Veterinária - UFMG - Belo Horizonte, MG \\ ${ }^{2}$ Departamento de Medicina Veterinária - UFLA - Lavras, MG \\ ${ }^{3}$ Faculdade de Medicina - UNESP - Botucatu, SP
}

A eletrocardiografia contínua (EC) é o método mais adequado para avaliar o ritmo cardíaco em cães, pois permite a avaliação durante 24 horas e a observação da atividade elétrica cardíaca com o animal em diferentes atividades (sono, exercício, refeição) e, consequentemente, em estados fisiológicos variados (Ware, 2007).

A EC é o exame de escolha na identificação de arritmias com sinais clínicos intermitentes, na identificação da gravidade da cardiomiopatia arritmogênica ventricular direita de cães e apresenta importância na predição e no prognóstico da cardiomiopatia dilatada (CMD) (Ware, 2007).

Os cães da raça Pastor Alemão podem apresentar cardiopatias (Ware, 2007) e morte súbita devido à arritmia familiar. Essa arritmia familiar não apresenta alteração necroscópica; embora mais presente em filhotes e jovens, pode ser detectada em cães com mais de cinco anos de idade. São considerados acometidos os animais que apresentem mais de 240 complexos ventriculares prematuros (CVP) durante o exame (Möise et al., 1997).

Em seres humanos, a atividade física está relacionada à modulação do sistema nervoso autônomo por aumento da atividade parassimpática ou diminuição da atividade simpática, principalmente no início do repouso (Martinelli et al., 2005). Além disso, a realização

Recebido em 7 de maio de 2009

Aceito em 20 de novembro de 2009

E-mail: guialbuquerque@yahoo.com

Apoio: FAPEMIG de atividade física é estimulante da regeneração hepática por diminuição da atividade simpática (Oben et al., 2003) e causa diminuição da pressão arterial sistêmica (Billman, 2002).

Como as cardiopatias podem cursar com alteração de ritmo e como não foi encontrado, na literatura pesquisada, estudo de EC em cães atletas, este trabalho teve o objetivo de avaliar o ritmo cardíaco à EC em cães da raça Pastor Alemão.

Foram utilizados 25 cães da raça Pastor Alemão, clinicamente saudáveis, 15 machos e 10 fêmeas, com idades entre sete meses e 10 anos (média de $3,24 \pm 2,24$ anos) e pesos entre 22 e $45 \mathrm{~kg}$ (média de $32,36 \pm 5,36 \mathrm{~kg}$ ), distribuídos em três grupos de acordo com a idade: A - nove animais com idades entre sete e 24 meses; $\mathrm{B}-12$ animais com idades entre 24 e 48 meses; $\mathrm{C}$ - quatro animais com idade acima de 48 meses. Os cães foram também diferenciados em dois grupos de acordo com a atividade física: 11 que realizavam atividade física regularmente (AFR) e 14 que não a realizavam (S). Foram considerados, como praticantes de atividades físicas regulares, os cães que caminhavam três vezes por semana, por $3 \mathrm{~km}$ em 30 minutos, há pelo menos três meses.

A eletrocardiografia contínua foi realizada com monitor Holter ${ }^{1}$ de dois canais. Os eletrodos foram fixados na pele da região torácica do animal, de acordo com Ware (2007), e as atividades do animal foram anotadas no horário do acontecimento, em formulário próprio.

${ }^{1}$ Rozinn Electronics Inc. (Holter recorder model 151) Glendale, NY, EUA. 
Avaliaram-se a frequência cardíaca máxima (FCmáx), a frequência cardíaca média (FCmd) e a frequência cardíaca mínima (FCmín), e estudaram-se os segmentos $\mathrm{ST}$, as pausas sinusais (PS) e a ocorrência de arritmias. Considerou-se pausa sinusal (PS) quando o período sem registro de P-QRS-T foi igual ou superior a 2 seg.

As FC foram avaliadas quanto à normalidade pelo teste de Kolmogorov-Smirnov. A influência do sexo, da atividade física regular e da idade nas FCmáx, FCmd e na FCmín foram avaliadas pela análise de variância por meio do programa SISVAR versão 4.6. A avaliação das arritmias, das alterações do segmento ST e das PS foi realizada de maneira descritiva, comparando-se com o formulário de anotações.

As FCmáx, FCmd e FCmín (média \pm desviopadrão) foram $212,20 \pm 40,32,101,40 \pm 33,50 \mathrm{e}$ $47,52 \pm 10,62$, respectivamente. Os valores de $\mathrm{P}$ para o sexo e para a idade na FCmáx, na FCmd e na FCmín foram 0,$80 ; 0,73 ; 0,24$ e 0,$18 ; 0,26$; 0,37 , respectivamente. Os resultados indicaram que não houve influência do sexo e da idade na FC; resultados semelhantes aos observados em humanos, pois as pessoas mais idosas não apresentam diferença nas FC em comparação com as mais novas, e não há diferença entre os sexos (Jensen-Urstad et al., 1997).

Em humanos, há diferença entre atletas e sedentários, porém não houve concordância entre os estudos que analisaram essa variabilidade da FC, se a diferença ocorre no sistema simpático ou parassimpático (Dixon et al., 1992; Furlan et al., 1993). A realização de atividade física, provavelmente, modula o nodo sinusal diminuindo a FCmd nos atletas (Martinelli et al., 2005).

Stepien et al. (1998) estudaram, por meio de ecocardiograma, cães do Alasca, antes e após treinamento de cinco meses, e verificaram mudanças nos padrões ecocardiográficos e diminuição da $\mathrm{FC}$ de $133 \pm 24$ para $113 \pm 23$. Neste experimento, os cães AFR apresentaram FCmd menores que os $\mathrm{S}(\mathrm{P}=0,03)$, mostrando que, semelhante ao que ocorre em humanos (Martinelli et al., 2005), a atividade física regular é benéfica ao funcionamento cardíaco dos cães.

Os AFR apresentaram FCmd de $80,89 \pm 13,85$ e os $\mathrm{S}$ de $112,94 \pm 35,71$, respectivamente. Os AFR e os $\mathrm{S}$ apresentaram semelhança no aumento e na diminuição da FCmd ao longo do dia, porém a diminuição na FC ocorreu de maneira mais rápida nos $\mathrm{AFR} \mathrm{e}$ foi verificada com mais evidência, a partir das 22 horas (Fig. 1). O P da FCmáx e o da FCmín na comparação dos grupos com diferentes atividades físicas foram de $0,06 \mathrm{e}$ de 0,65 , respectivamente, mostrando maior tendência de igualdade nas FCmín entre os animais AFR e $\mathrm{S}$.

A EC detecta arritmias ventriculares e alterações no segmento ST. Estes achados ocorrem na forma grave de estenose subaórtica, doença congênita comum em cães da raça Pastor Alemão (Ware, 2007). Não foram observadas elevações nem depressões patológicas do segmento ST em nenhum dos 25 cães estudados. Este resultado está de acordo com a afirmação da literatura especializada, segundo a qual cães sadios dificilmente apresentam alteração de segmento ST à EC (Ware, 2007).

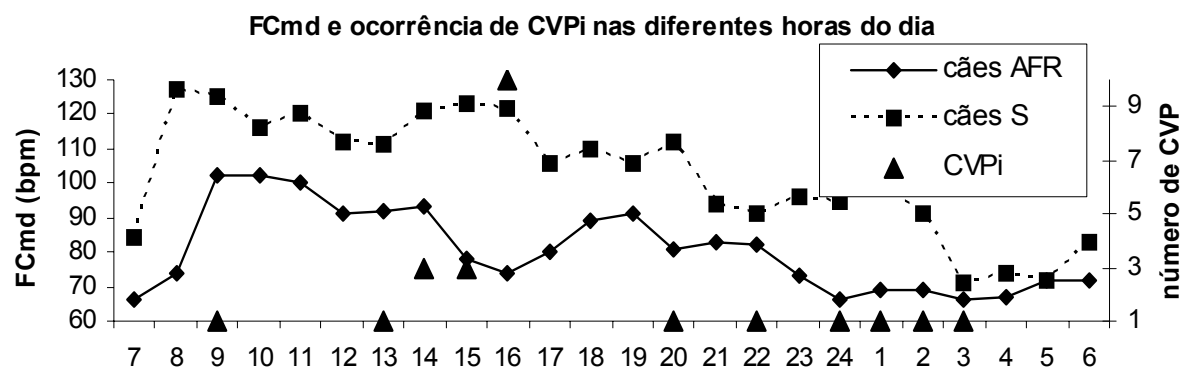

horas do dia

Figura 1. Médias das frequências cardíacas médias (FCmd) e ocorrência de complexos ventriculares prematuros isolados (CVPi) nas diferentes horas do dia em cães da raça Pastor Alemão, praticantes de atividade física regular (AFR) e não praticantes (S). 


\section{Cavalcanti et al.}

As arritmias atriais ocorrem principalmente nos casos de grande dilatação atrial secundária à CMD e à insuficiência das valvas atrioventriculares (Ware, 2007). A presença de arrtimias atriais, geralmente, é observada em menos de $25 \%$ dos animais sadios (Leomil Neto et al., 2002; Nogueira et al., 2006). Neste trabalho, nenhum cão apresentou arritmia atrial.

A PS longa ou em grande frequência é um fator de instauração de arritmias (Ware, 2007). Möise et al. (1997) mostraram que, geralmente, após os momentos de maior intervalo entre as ondas $\mathrm{R}$ é que ocorrem as taquicardias ventriculares graves em cães Pastores Alemães jovens acometidos da morte súbita familiar.

Observaram-se PS em 10 animais (40\%), sendo a maioria detectada no momento de menor FC, geralmente à noite, quando os animais se encontravam tranquilos ou adormecidos. A ocorrência de PS foi semelhante entre os grupos AFR e S, $36 \%$ e $43 \%$, respectivamente. Leomil Neto et al. (2002) observaram PS em todos os cães saudáveis das raças Dobermann e Boxer, e em $70 \%$ da raça Cocker Spaniel. Isto mostra que o número de cães saudáveis que apresentam PS à $\mathrm{EC}$ varia entre as raças.

A mediana de ocorrência das PS foi de 18 vezes, com média da maior pausa de $2,87 \pm 0,58 \mathrm{seg}$ e coeficiente de variação de $140 \%$, com ocorrência de dois a 204 episódios de PS em 24 horas. Comparando-se com cães das raças Cocker Spaniel, Doberman e Boxer (Leomil Neto et al., 2002), percebe-se que o valor da mediana das PS varia mais entre as raças do que os valores dos maiores intervalos entre as ondas $\mathrm{R}$.

A ocorrência de arritmias ventriculares em cães saudáveis, durante 24 horas, varia entre as raças. Foram relatadas ocorrências em $26 \%$ dos cães da raça Beagle (Yamada e Tokuriki, 2000), em 10\% da raça Dobermann, em $40 \%$ da raça Cocker Spaniel (Leomil Neto et al., 2002) e em 50 e $70 \%$ da raça Boxer (Leomil Neto et al., 2002; Nogueira et al., 2006).

Neste trabalho, 20\% (5/25) dos cães apresentaram complexos ventriculares prematuros isolados (CVPi), sendo que, nos momentos de intenso estímulo parassimpático, entre 3 e 5h, não houve registro de CPV (Fig. 1). Portanto, grandes intervalos entre as ondas $\mathrm{R}$ não estimularam os CVPi nos animais deste estudo, mas, provavelmente, o início do aumento da estimulação parassimpática juntamente com a diminuição da atividade simpática causaram a maior ocorrência dos CVPi, entre 22 e $3 \mathrm{~h}$ da manhã.

A maior ocorrência de CVPi às $16 \mathrm{~h}$ é atribuída à atividade ectópica de um único animal AFR. Quanto à atividade física, três animais do grupo $\mathrm{S}$ apresentaram CVPi, com dois, dois e três episódios em 24 horas, e dois do AFR também apresentaram CVPi; neste grupo, um animal apresentou um e o outro 18 episódios.

Considerando a manifestação espontânea de CVPi em cães de diferentes raças, assim como a baixa ocorrência registrada em 24 horas nos grupos com diferentes níveis de atividade física estudados, a presença de CVPi não foi associada ao sedentarismo. Em humanos, os atletas de alto desempenho apresentam menos episódios de taquicardia ventricular que os indivíduos sedentários (Billman, 2002).

Em cães Pastores Alemães jovens, já se registrou morte súbita familiar relacionada à presença de taquicardias ventriculares, principalmente nos momentos de maior influência parassimpática, como durante o sono profundo ou depois de exercício (Möise et al., 1997).

No grupo A deste trabalho, apenas uma cadela de dois anos de idade apresentou um CVPi, que ocorreu às 13h, em momento de FCmd 127bpm, muito próxima da FCmd do próprio animal (125bpm), portanto não foi encontrada qualquer evidência da presença dessa doença entre os cães estudados.

Os resultados obtidos poderão auxiliar na avaliação da EC de cães da raça Pastor Alemão e mostraram que a realização de exercício físico pode modular a atividade do nodo sinusal em cães.

Palavras-chave: cão, Pastor Alemão, eletrocardiografia contínua, atividade física 


\begin{abstract}
The pattern for ambulatory electrocardiography was evaluated in 25 healthy German Shepherd dogs. The influences of gender, age, and physical activity in HR maximum, mean, and minimum (HRmx, HRme, and HRmin, respectively) were studied. The physically active dogs (PA) showed lower HRme than sedentary animals $(S)(P=0.03)$, whereas HRmx and HRmin were not altered ( $P=0.06$ and $P=0.65$, respectively).

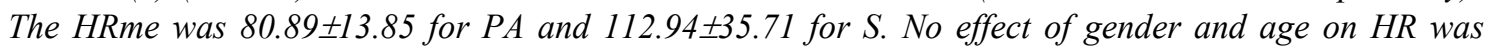
observed. It is possible to state that the physical activity can modulate the sinus node of the dogs.
\end{abstract}

Keywords: dog, German Shepherd, ambulatory electrocardiography, physical activity

\section{REFERÊNCIAS BIBLIOGRÁFICAS}

BILLMAN, G.E. Aerobic exercise conditioning: a nonpharmacological antiarrhythmic intervention. J. Appl. Physiol., v.92, p.446-454, 2002.

DIXON, E.M.; KAMATH, M.V.; MCCARTNEY, N. et al. Neural regulation of heart rate variability in endurance athletes and sedentary controls. Cardiovasc. Res., v.26, p.713-719, 1992.

FURLAN, R.; PIAZZA, S.; DELL'ORTO, S. et al. Early and late effects of exercise and athletic training on neural mechanisms controlling heart rate. Cardiovasc. Res., v.27, p.482-488, 1993.

JENSEN-URSTAD, K.; STORCK, N.;

BOUVIER, F. et al. Heart rate variability in healthy subjects is related to age and gender. Acta Physiol. Scand., v.160, p.235-241, 1997.

LEOMIL NETO, M.; LARSSON, M.H.M.A.; PEREIRA, L. et al. Padronização da monitorização eletrocardiográfica por 24 horas em cães. Arq. Bras. Med. Vet. Zootec., v.54, p.133-138, 2002.

MARTINELLI, F.S.; CHACON-MIKAHIL, M.P.T.; MARTINS, L.E.B. et al. Heart rate variability in athletes and nonathletes at rest and during head-up tilt. Braz. J. Med. Biol. Res., v.38, p.639-647, 2005.
MÖISE, N.S.; GILMOUR, R.F.; RICCIO, M.L. et al. Diagnosis of inherited ventricular tachycardia in German Shepherd dogs. J. Am. Vet. Med. Assoc., v.210, p.403-410, 1997.

NOGUEIRA, R.B.; MUZZI, R.A.L.; HERRERA, D.S. et al. Avaliação do ritmo cardíaco em cães da raça boxer saudáveis por meio da eletrocardiografia contínua (holter). Arq. Bras. Med. Vet. Zootec., v.58, p.133-136, 2006.

OBEN, J.A.; ROSKAMS, T.; YANG, S. et al. Sympathetic nervous system inhibition increases hepatic progenitors and reduces liver injury. Hepatology, v.38, p.664-673, 2003.

STEPIEN, R.L.; HINCHCLIFF, K.W.; CONSTABLE, P.D. et al. Effect of endurance training on cardiac morphology in Alaskan sled dog. J. Appl. Physiol., v.85, p.1368-1375, 1998.

YAMADA, M.; TOKURIKI, M. Effects of a canine Elizabethan collar on ambulatory electrocardiogram recorded by a holter recording system and spontaneous activities measured continuously by an accelerometer in beagle dogs. J. Vet. Med. Sci., v.62, p.549-552, 2000.

WARE, W.A. (Ed). Cardiovascular disease in small animal medicine. London: Manson, 2007. 396p. 